\title{
Cutaneous epithelioid hemangioma mimicking infected Montgomery tubercle
}

\section{Padmapriya Jaiprakash, Kanthilatha Pai, Vidya Monappa}

\author{
Department of Pathology, Kasturba Medical College, Manipal Academy of Higher Education, Manipal, India
}

Corresponding author: Dr. Padmapriya Jaiprakash, E-mail: padmapriya.j@gmail.com

\begin{abstract}
Cutaneous epithelioid hemangioma is a vascular lesion of uncertain pathogenesis. Recurrences are common in cases with incomplete surgical excision. Histologically, it can be differentiated from other conditions by the presence of prominent endothelial lining and mixed inflammatory infiltrate in the background with predominance of lymphocytes and eosinophils. A 19 year old lady presented with a painful left breast swelling, which was clinically diagnosed as infected Montgomery's tubercle and excised. Histopathology showed features of cutaneous epithelioid hemangioma. Cutaneous epithelioid hemangioma can occur rarely in the breast, where it can mimic an inflammatory pathology.
\end{abstract}

Key words: Epithelioid; Hemangioma; Breast

\section{INTRODUCTION}

Cutaneous epithelioid hemangioma, also known as angiolymphoid hyperplasia with eosinophilia (ALHE), is an entity whose origin is controversial. It is said to be a neoplastic process or a reactive proliferation secondary to various stimuli, including trauma [1]. Most involve dermis, subcutaneous or deeper tissues. Sites commonly involved include external ear, occipital region and around temporal artery. Deeper tissues include head and neck region, arm, hands, axillae and inguinal region. Rare sites including oral cavity, tongue, lymph node, bone, testis and breast have been reported [2].

\section{CASE REPORT}

A 19 year old lady presented to the OPD with a pruritic and painful lesion in the left breast since 1 month. There was no history of similar lesions in the past or any other parts of the body. On examination, a single lesion was noted in the areola of the left breast with local raise in temperature. A diagnosis of infected Montgomery's tubercle was made and an excision biopsy done. The sections studied showed proliferation of small sized blood vessels (Fig. 1) with vague lobular architecture, lined by plump (epithelioid) endothelial cells (Fig. 2) surrounded by dense perivascular inflammatory infiltrate composed of lymphocytes and eosinophils (Fig. 3). A diagnosis of epithelioid hemangioma or ALHE was made. Reticulin stain done highlighted the vascular channels (Fig. 4). On follow-up, the patient was asymptomatic. Prior to the study, patient gave written consent to the examination and biopsy after having been informed about the procedure.

\section{DISCUSSION}

Epithelioid hemangioma affects females more than males and most commonly involves pre-auricular area and scalp. Systemic eosinophilia is seen in $20 \%$ of cases [2]. Grossly, they are circumscribed lesions measuring 0.5 to $2 \mathrm{cms}$ in size. Epithelioid hemangioma are characterized by a prominent proliferation of small, capillary-sized vessels lined by plump, epithelioid endothelial cells. The vessels typically have an immature appearance. Early lesions demonstrate a predominance of rapidly proliferating atypical vasculature [3]. Late lesions illustrate maturation of these blood vessels with prevalence of lymphoid follicles seen towards the

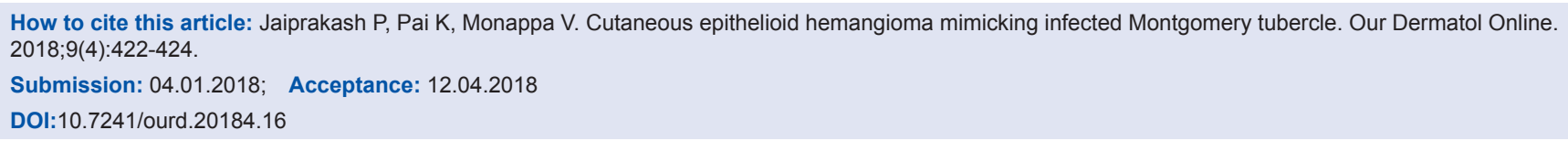




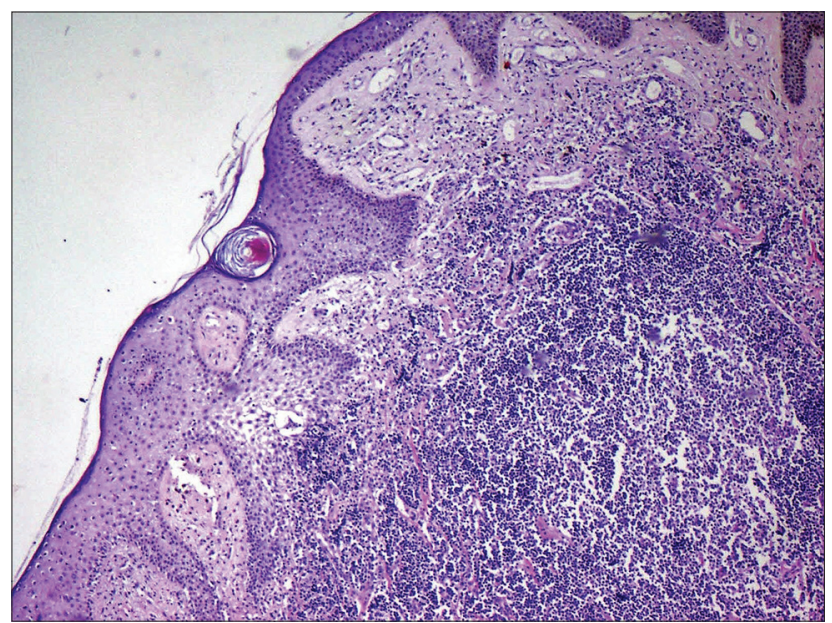

Figure 1: Epidermis overlying dermis showing a lesion composed of vascular proliferation surrounded by lymphocytic infiltrate with few eosinophils (H\&E, 40x).

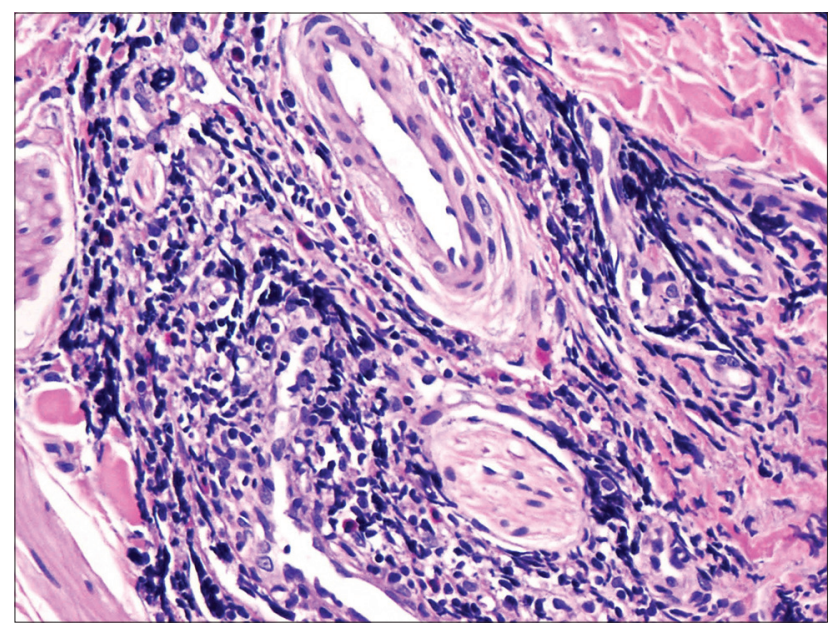

Figure 2: Vascular channels lined by plump epithelioid cells, surrounded by lymphocytes with few eosinophils (H\&E, 100x).

periphery of the lesion. Complete local excision and follow-up are optimal management for epithelioid hemangioma. Local recurrence is reported to occur in up to one-third of patients.

Amongst the differential diagnosis, first is Kimura disease $[3,4]$. Though used as synonyms previously, Kimura disease typically presents as a subcutaneous nodule in young male, in the preauricular or submandibular region. ${ }^{1}$ Microscopically, it has characteristic eosinophilic microabscesses and lacks epithelioid cells seen in ALHE. The other ominous differentials include epithelioid angiosarcoma and epithelioid hemangioendothelioma (EHE). Angiosarcoma shows obvious malignant nuclear features, along with anastomosing vascular channels. EHE shows the presence of cords of vacuolated

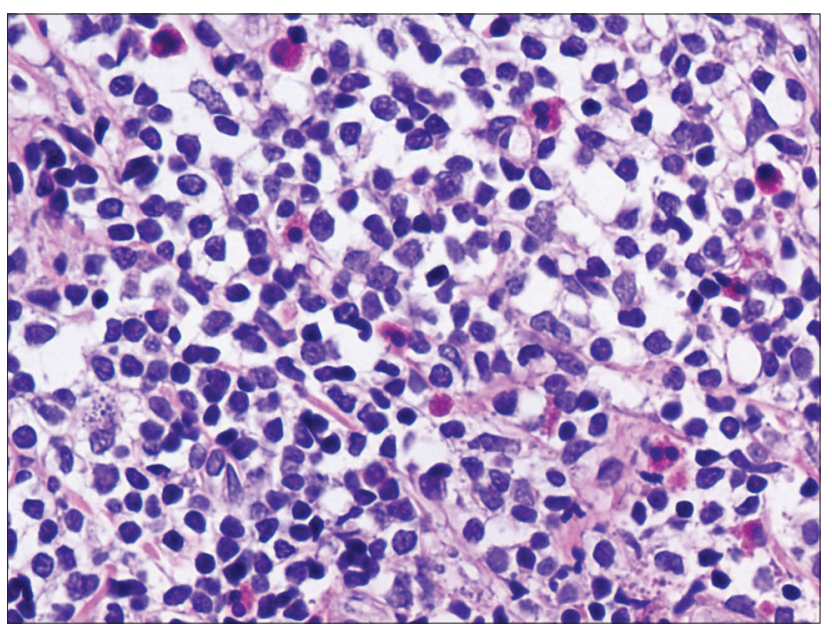

Figure 3: Polymorphous population of lymphocytes with interspersed eosinophils (H\&E, 200x).

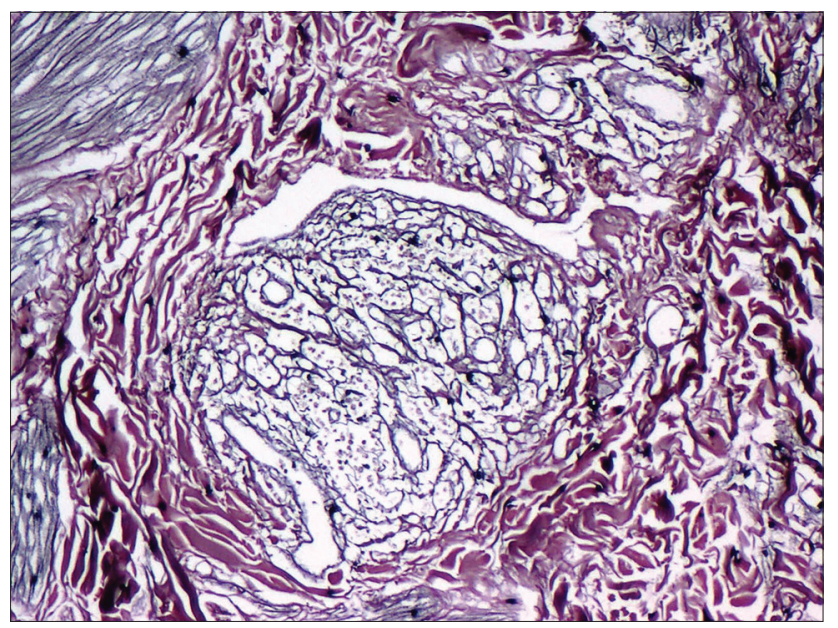

Figure 4: Reticulin fibres highlighting the vascular channels surrounded by the lymphocytes (Reticulin stain, 100x).

endothelial cells in a myxoid matrix, along with the absence of the lymphoid aggregates [5].

Clinical differentials include Kaposi sarcoma and pyogenic granuloma, both of which show characteristic vascular channels and are not microscopic mimics [5].

\section{CONCLUSION}

This case is being presented for involvement of a rare site, mimicking an infective lesion.

\section{ACKNOWLEDGEMENTS}

We would like to acknowledge the technical team of Histopathology Lab of Kasturba Medical College, 
Manipal, India and Dr. Y. S. Rao, Consultant surgeon in Udupi for providing us with the clinical details.

\section{Consent}

The examination of the patient was conducted according to the Declaration of Helsinki principles.

\section{REFERENCES}

1. Guo R, Gavino AP. Angiolymphoid Hyperplasia With Eosinophilia. Arch Pathol Lab. 2015;139:683-6.

2. Gupta M. Angiolymphoid hyperplasia with eosinophilia - A report of three cases. Our Dermatol Online. 2018;9:167-9.

3. Brodie C, Provenzano E. Vascular proliferations of the breast. Histopathology. 2008;52:30-44

4. Park SY, Lee JK, JO S, Huh CH, Cho KH, NA JI. Cutaneous epithelioid hemangioendothelioma presented as an ulcerated areolar mass. J Dermatol. 2014;41:112-3.

5. Tirumalasetti N. Angiolymphoid hyperplasia with eosinophilia: A rare benign vascular tumor of breast. Indian J Pathol Microbiol. 2013;56:405-7.

Copyright by Padmapriya Jaiprakash, et al. This is an open access article distributed under the terms of the Creative Commons Attribution License, which permits unrestricted use, distribution, and reproduction in any medium, provided the original author and source are credited.

Source of Support: Nil, Conflict of Interest: None declared. 